\title{
On a semilinear elliptic equation with inverse-square potential
}

\author{
Haïm Brezis * ${ }^{\dagger}$, Louis Dupaigne * and Alberto Tesei ${ }^{\ddagger}$
}

In this paper we study existence and nonexistence of solutions $u \geq 0$ of the equation :

$$
-\Delta u=\frac{c}{r^{2}} u+u^{p}
$$

in a ball $B(0, R)$ of $\mathbb{R}^{N}, N \geq 3$. Here $r=|x|, p>1$ and the coefficient $c$ satisfies the inequality $0<c \leq c_{0}$, where $c_{0}=(N-2)^{2} / 4$ is the best constant in the Hardy inequality.

In this study an important role is played by the roots

$$
\alpha=\alpha^{ \pm}:=(N-2) / 2 \pm \sqrt{c_{0}-c}
$$

of the equation

$$
\alpha^{2}-(N-2) \alpha+c=0 .
$$

Observe that $\alpha^{+}>\alpha^{-}>0$.

Our main result asserts that nontrivial solutions of equation (1) exist if and only if $p<p^{+}$where

$$
p^{+}=1+2 / \alpha^{-} .
$$

Theorem 1. Let $0 \leq c \leq c_{0}$. For any $p \in\left(1, p^{+}\right)$, there exists a nontrivial solution to equation (1) with $u^{p}$ and $u / r^{2}$ belonging to $L^{1}\left(B_{R}\right)$ and (1) holds in $\mathcal{D}^{\prime}\left(B_{R}\right)$.

The proof of Theorem 1 is straightforward and elementary, except for the limiting value $c=c_{0}$. The conclusion of Theorem 1 was known in many-but presumably not all- cases (see e.g. [12]). Concerning nonexistence we have

Theorem 2. Let $0<c \leq c_{0}, p \geq p^{+}$. Assume $u \in L_{\text {loc }}^{p}\left(B_{R} \backslash\{0\}\right), u \geq 0$ satisfies

$$
-\Delta u-\frac{c}{r^{2}} u \geq u^{p}
$$

in $\mathcal{D}^{\prime}\left(B_{R} \backslash\{0\}\right)$. Then $u \equiv 0$.

\footnotetext{
* Laboratoire Jacques-Louis Lions, Université Pierre et Marie Curie, Boîte Courrier 187, 4 place Jussieu, 75252 Paris Cedex 05, France.

brezis@ann.jussieu.fr, dupaigne@ann.jussieu.fr

$\dagger$ Department of Mathematics - Hill Center, Rutgers Univ., 110 Frelinghuysen Rd., Piscataway, NJ 08854-8019 USA.

brezis@math.rutgers.edu

$\ddagger$ Dipartimento di Matematica "G. Castelnuovo", Università di Roma "La Sapienza", P.le A. Moro 5, I-00185 Roma, Italia.

tesei@mat.uniroma1.it
} 
Theorem 2 is reminiscent of the nonexistence results of Brezis-Cabre [2] concerning the so-called very weak solutions to the inequality

$$
-\Delta u \geq \frac{u^{p}}{r^{2}}, \quad u \geq 0, \quad u \in L_{l o c}^{p}\left(B_{R} \backslash\{0\}\right),
$$

for any $p>1$. The nonexistence aspect in (1) when $p \geq p^{+}$was first investigated by Pohozaev-Tesei [11]. However the concept of solution used there was stronger; our concept is the weakest possible.

We also observe that Theorem 2 seems (formally) to contradict the Implicit Function Theorem since there is no solution of $-\Delta u=\left(c /|x|^{2}\right) u+u^{p}+t$, even when $t>0$ is small. As observed in [1], this is due to the lack of an appropriate functional space in which to apply the IFT.

\section{Proof of Theorem 1.}

Set $p^{-}=1+2 / \alpha^{+}$and observe that

$$
\begin{gathered}
1<p^{-}<\frac{N+2}{N-2}<p^{+} \quad \text { for any } 0<c<c_{0} \text { and } \\
\lim _{c \rightarrow 0} p^{-}=\frac{N}{N-2}, \quad \lim _{c \rightarrow c_{0}} p^{-}=\frac{N+2}{N-2}, \\
\lim _{c \rightarrow 0} p^{+}=+\infty, \quad \lim _{c \rightarrow c_{0}} p^{+}=\frac{N+2}{N-2} .
\end{gathered}
$$

We distinguish three cases :

$$
\text { Case } 1: 0 \leq \mathrm{c}<\mathrm{c}_{0} \text { and } \mathrm{p}<\frac{\mathrm{N}+2}{\mathrm{~N}-2} \text {. }
$$

Here the existence of a positive solution $u \in H_{0}^{1}\left(B_{R}\right)$ of (1) is a standard and straightforward consequence of the Mountain Pass Theorem. In fact, one can find a radial solution by working in the class of radial functions.

Case $2: 0 \leq \mathrm{c}<\mathrm{c}_{0}$ and $\mathrm{p}^{-}<\mathrm{p}<\mathrm{p}^{+}$.

Here we have an explicit solution of (1) of the form $u=A / r^{\beta}$ with $\beta=2 /(p-1)$, $A>0$ given by

$$
A^{p-1}=-\beta^{2}+(N-2) \beta-c>0,
$$

because $\alpha^{-}, \alpha^{+}$are the roots of (2) and the restriction $\alpha^{-}<\beta<\alpha^{+}$is equivalent to the condition $p^{-}<p<p^{+}$. Since $\beta<N-2$, u satisfies (1) in the sense of $\mathcal{D}^{\prime}\left(B_{R}\right)$. 
Case 3: $\mathrm{c}=\mathrm{c}_{0}$ and $1<\mathrm{p}<\mathrm{p}^{+}=\frac{\mathrm{N}+2}{\mathrm{~N}-2}$.

This case is a little more delicate : here we need the improved Hardy inequality which asserts that

$$
\int_{B_{R}}|\nabla u|^{2} \geq c_{0} \int_{B_{R}} \frac{u^{2}}{r^{2}}+c_{q}\|u\|_{L^{q}\left(B_{R}\right)}^{2},
$$

for any $1 \leq q<\frac{2 N}{N-2}$ and $u \in C_{0}^{\infty}\left(B_{R}\right)$. See [5]. Let $H$ be the Hilbert space obtained by completing $C_{0}^{\infty}\left(B_{R}\right)$ with respect to the scalar product

$$
a(u, v)=\int_{B_{R}} \nabla u \cdot \nabla v-c_{0} \int_{B_{R}} u v / r^{2}
$$

Clearly $H$ is contained in every $L^{q}\left(B_{R}\right)$ with $1 \leq q<\frac{2 N}{N-2}$ with continuous injection. Moreover the injection is compact. This fact is due to $\mathrm{H}$. Brezis and the proof is presented in Lemmas 3.2, 3.3 of [7]. We may then use the Mountain Pass Theorem in $H$ and the (PS) condition is satisfied.

\section{Proof of Theorem 2.}

We will use the following lemma :

Lemma 1. Let $\Sigma \subset \subset \Omega$ be a closed set of zero (newtonian) capacity and assume that $u, f \in$ $L_{l o c}^{1}(\Omega \backslash \Sigma)$ are two nonnegative functions such that

$$
-\Delta u \geq f \quad \text { in } \mathcal{D}^{\prime}(\Omega \backslash \Sigma)
$$

Then $u, f \in L_{l o c}^{1}(\Omega)$ and

$$
-\Delta u \geq f \quad \text { in } \mathcal{D}^{\prime}(\Omega)
$$

Furthermore given any smooth subdomain $\Omega^{\prime} \subset \subset \Omega$, if $v \in L^{1}\left(\Omega^{\prime}\right)$ is the solution of

$$
\left\{\begin{aligned}
-\Delta v=f & \text { in } \Omega^{\prime} \\
v=0 & \text { on } \partial \Omega^{\prime}
\end{aligned}\right.
$$

in the sense that

$$
\int v(-\Delta \phi)=\int f \phi \quad \forall \phi \in C^{2}\left(\bar{\Omega}^{\prime}\right) \quad \text { such that }\left.\quad \phi\right|_{\partial \Omega^{\prime}} \equiv 0,
$$

then

$$
u \geq v \quad \text { a.e. in } \Omega^{\prime}
$$




\section{Proof of Lemma 1}

This lemma can be seen as a fairly easy consequence of Theorem 7.7 in [9]. It is also closely related to a result in [4]. We provide a proof for completeness. Let $u_{k}=\min (u, k)$, $k>0$, which by Kato's Lemma (see [10]) satisfies

$$
-\Delta u_{k} \geq f_{k} \quad \text { in } \mathcal{D}^{\prime}(\Omega \backslash \Sigma),
$$

where $f_{k}:=f \chi_{\{u<k\}}$. Since $-\Delta u_{k}$ is a nonnegative distribution on $\Omega \backslash \Sigma$, it extends to a nonnegative measure on $\Omega \backslash \Sigma$. Since $u_{k}$ is bounded, it follows from a GagliardoNirenberg-type inequality that $u_{k} \in H_{l o c}^{1}(\Omega \backslash \Sigma)$. We show next that in fact $u_{k} \in H_{l o c}^{1}(\Omega)$. We first take a nonnegative function $\phi \in C_{0}^{\infty}(\Omega)$ and a sequence $\phi_{n} \in C_{0}^{\infty}(\Omega \backslash \Sigma)$ converging to $\phi$ in $H^{1}(\Omega)$. This is always possible since $\operatorname{cap}_{\Omega}(\Sigma)=0$ (take e.g. $\phi_{n}=\phi\left(1-\chi_{n}\right)$ where $\chi_{n}=1$ near $\Sigma$ and $\left.\left\|\chi_{n}\right\|_{H^{1}} \rightarrow 0=\operatorname{cap}_{\Omega}(\Sigma)\right)$. We then have, with $C_{k}=e^{k}$,

$$
\begin{aligned}
\int\left|\nabla u_{k}\right|^{2} \phi_{n}^{2} & \leq C_{k} \int e^{-u_{k}}\left|\nabla u_{k}\right|^{2} \phi_{n}^{2}=-C_{k} \int \phi_{n}^{2} \nabla\left(e^{-u_{k}}\right) \cdot \nabla u_{k} \\
& =C_{k}\left(2 \int e^{-u_{k}} \phi_{n} \nabla \phi_{n} \cdot \nabla u_{k}+\int e^{-u_{k}} \Delta u_{k} \phi_{n}^{2}\right) \\
& \leq 2 C_{k}^{2} \int e^{-u_{k}}\left|\nabla \phi_{n}\right|^{2}+\frac{1}{2} \int e^{-u_{k}}\left|\nabla u_{k}\right|^{2} \phi_{n}^{2},
\end{aligned}
$$

so that

$$
\int\left|\nabla\left(u_{k} \phi_{n}\right)\right|^{2} \leq C_{k}^{\prime} \int\left|\nabla \phi_{n}\right|^{2}
$$

Passing to the limit as $n \rightarrow \infty$ in the above inequality implies that $u_{k} \in H_{l o c}^{1}(\Omega)$.

We next show that

$$
-\Delta u_{k} \geq f_{k} \quad \text { in } \mathcal{D}^{\prime}(\Omega) \text {. }
$$

Take $\phi$ and $\phi_{n}$ as above so that by (4),

$$
\int u_{k}\left(-\Delta \phi_{n}\right) \geq \int f_{k} \phi_{n}
$$

Now, as $n \rightarrow \infty$,

$$
\int u_{k}\left(-\Delta \phi_{n}\right)=\int \nabla u_{k} \nabla \phi_{n} \rightarrow \int \nabla u_{k} \nabla \phi=-\int u_{k} \Delta \phi .
$$

Passing to the limit in (6) as $n \rightarrow \infty$, we thus obtain (5).

In particular $u_{k}$ is superharmonic in $\Omega$ and given almost any $x \in \Omega$ and any ball $B \subset \Omega$ centered at $x$, we have

$$
u_{k}(x) \geq \frac{1}{|B|} \int_{B} u_{k}(y) d y .
$$

Now, since $u \in L_{l o c}^{1}(\Omega \backslash \Sigma)$ (and $\left.|\Sigma|=0\right), u_{k} \rightarrow u$ a.e. in $\Omega$ as $k \rightarrow \infty$ and $u$ is finite almost everywhere. By Fatou's Lemma we then conclude from (7) that for almost every ball $B$, 


$$
\int_{B} u<\infty
$$

which means that $u \in L_{l o c}^{1}(\Omega)$. Using this information, we can now easily pass to the limit in (5) and conclude that $f \in L_{l o c}^{1}(\Omega)$ and that

$$
-\Delta u \geq f \quad \text { in } \mathcal{D}^{\prime}(\Omega) \text {. }
$$

It only remains to prove (3). We let $\rho_{n}$ be a standard smooth mollifier and let $u_{n}=$ $u * \rho_{n}, f_{n}=f * \rho_{n}$ so that for $n$ large enough $-\Delta u_{n} \geq f_{n}$ and $u_{n} \geq 0$ in $\Omega^{\prime}$. By the Maximum Principle

$$
u_{n} \geq v_{n} \quad \text { in } \Omega^{\prime}
$$

where $v_{n}$ solves

$$
\left\{\begin{aligned}
-\Delta v_{n} & =f_{n} \quad \text { in } \Omega^{\prime} \\
v_{n} & =0 \quad \text { on } \partial \Omega^{\prime}
\end{aligned}\right.
$$

As $n \rightarrow \infty, u_{n} \rightarrow u$ in $L^{1}\left(\Omega^{\prime}\right), f_{n} \rightarrow f$ in $L^{1}\left(\Omega^{\prime}\right)$ and (by Lemma 4 in [3]) $v_{n} \rightarrow v$ in $L^{1}\left(\Omega^{\prime}\right)$, which yields the desired conclusion.

\section{Proof of Theorem 2}

We argue by contradiction and assume that $u \not \equiv 0$. By Lemma $1, u \in L_{l o c}^{p}\left(B_{R}\right)$, $u / r^{2} \in L_{l o c}^{1}\left(B_{R}\right)$ and by the mean-value formula for superharmonic functions, given $R^{\prime} \in(0, R)$, there exists $\epsilon>0$ such that $u \geq \epsilon$ a.e. in $B_{R^{\prime}}$. Let $\lambda:=\epsilon^{q-1} / 2>0$ and $v_{0}$ be the solution of

$$
\left\{\begin{aligned}
-\Delta v_{0}=\lambda & \text { in } B_{R^{\prime}} \\
v_{0}=0 & \text { on } \partial B_{R^{\prime}} .
\end{aligned}\right.
$$

Once more by Lemma 1, we have

$$
0 \leq v_{0} \leq u
$$

Next, for $n \geq 1$, define inductively $v_{n}$ by

$$
\left\{\begin{aligned}
-\Delta v_{n} & =\frac{c}{|x|^{2}} v_{n-1}+\frac{1}{2} v_{n-1}^{p}+\lambda & & \text { in } B_{R^{\prime}} \\
v_{n} & =0 & & \text { on } \partial B_{R^{\prime}} .
\end{aligned}\right.
$$

In order to have a well-defined solution $v_{n}$ (in the sense of Lemma 4 in [3]) it suffices to prove that $f:=\frac{c}{|x|^{2}} v_{n-1}+\frac{1}{2} v_{n-1}^{p} \in L^{1}\left(B_{R^{\prime}}\right)$. When $n=0$, this follows from (8) and Lemma 1 which implies that $\frac{c}{|x|^{2}} u+\frac{1}{2} u^{p} \in L^{1}\left(B_{R^{\prime}}\right)$. Assume now that $v_{n-1} \in L^{1}\left(B_{R^{\prime}}\right)$ is well-defined. Using the Maximum Principle, it is easy to see that

$$
0 \leq v_{0} \leq v_{1} \leq \cdots \leq v_{n-1} \leq u
$$

whence $f \in L^{1}\left(B_{R^{\prime}}\right)$ and by the Maximum Principle again $0 \leq v_{n-1} \leq v_{n} \leq u$. 
By monotone convergence, letting $v:=\lim _{n \rightarrow \infty} v_{n}$, we have that

$$
\left\{\begin{aligned}
-\Delta v & =\frac{c}{|x|^{2}} v+\frac{1}{2} v^{p}+\lambda \quad \text { in } B_{R^{\prime}} \\
v & =0 \text { on } \partial B_{R^{\prime}},
\end{aligned}\right.
$$

in the sense that given any $\phi \in C^{2}\left(\bar{B}_{R^{\prime}}\right)$ such that $\left.\phi\right|_{\partial B_{R^{\prime}}} \equiv 0$,

$$
\int v(-\Delta \phi)=\int \frac{c}{|x|^{2}} v \phi+\frac{1}{2} \int v^{p} \phi+\lambda \int \phi .
$$

This contradicts Theorem 1 of [6].

Remark 1. Theorems 1 and 2 extend to more general situations-for example, when $u^{p}$ is replaced by $|x|^{-\beta} u^{q}$. Assume $0<c \leq c_{0}$ and set $q^{+}=1+\frac{2-\beta}{\alpha^{-}}$, where $\alpha^{-}$is as above. The conclusions of Theorems 1 and 2 remain valid with $p^{+}$replaced by $q^{+}$.

Remark 2. The argument presented in the proof of Theorem 2 may be used to provide a slightly simpler proof of Theorem 1 in [2].

Remark 3. Theorem 2 can be extended to problems of the type

$$
-\Delta u=\frac{c}{\operatorname{dist}(x, \Sigma)^{2}} u+u^{p},
$$

where $c>0$ is a small constant, $\Sigma$ is a smooth compact manifold of codimension $k \geq 3$ and $p$ is larger than some critical exponent, which can be computed explicitly in terms of $k$ and $c$. The argument is the same as in the proof of Theorem 2 except that the result of [6] is replaced by a result from [8].

Acknowledgments. The three authors are partially supported by an EC Grant through the RTN Program "Fronts-Singularities", HPRN-CT-2002-00274. The first author (H.B.) is also a member of the Institut Universitaire de France.

\section{References}

[1] H. Brezis, Is there failure of the Inverse Function Theorem ?, Proceedings of the Workshop held at the Morningside Center of Mathematics, Chinese Academy of Science, Beijing, June 1999.

[2] H. Brezis and X. Cabré, Some simple nonlinear PDE's without solutions, Bull UMI, 1 (1998), 223-262.

[3] H. Brezis, X. Cabré, Y. Martel and A. Ramiandrisoa, Blow-up for $u_{t}-\Delta u=g(u)$ revisited, Adv. Diff. Eq., 1 (1996), 73-90. 
[4] H. Brezis and P.L. Lions, A note on isolated singularities for linear elliptic equations, Mathematical analysis and applications, Adv. in Math. Suppl. Stud., 7a, Academic Press, New York-London, 1981, 263-266.

[5] H. Brezis and J.L. Vazquez, Blow-up solutions of some nonlinear elliptic problems, Rev. Mat. Univ. Complut. Madrid, 10 (1997), 443-469.

[6] L. Dupaigne, A nonlinear elliptic PDE with the inverse-square potential, J. d'Analyse Mathématique, 86 (2002), 359-398.

[7] J. Dávila and L. Dupaigne, Comparison principles for PDE's with a singular potential, Proc. Roy. Soc. Edinburgh, 133A (2003), 61-83.

[8] L. Dupaigne and G. Nedev, Semilinear elliptic PDE's with a singular potential, Adv. Differential Equations, 7 (2002), 973-1002.

[9] L.L. Helms, Introduction to potential theory, New York, Wiley and Sons, 1969.

[10] T. Kato, Schrödinger operators with singular potentials, Israel J. Math., 13 (1972), 135148.

[11] S.I. Pohozaev and A. Tesei, Nonexistence of local solutions to semilinear partial differential inequalities, Nota Scientifica 01/28, Dip. Mat. Universitá "La Sapienza", Roma (2001).

[12] S. Terracini, On positive entire solutions to a class of equations with a singular coefficient and critical exponent, Adv. Differential Equations, 1 (1996), 241-264. 